\title{
Prenatal Dental Development as a Reference Standard for Embryologic Status
}

\author{
STANLEY M. GARN, ALPHONSE R. BURDI, RICHARD L. MILLER, and \\ JERROLD M. NAGY \\ Center for Human Growth and Development and Department of Anatomy, University of \\ Michigan, Ann Arbor, Michigan 48104
}

Postnatal dental development may be used as a reference standard in the absence of exact-age information because dental variability is less than variability for other calcified structures (S. M. GARN, A. B. LEWIS, and D. L. PolACHECK, Science 128:1510, 1958; J Dent Res 38: $135-148,1959$ ) and is not affected much by nutritional status (S. M. GARN, A. B. LEWIS and R. S. KEREWSKY, J Dent Res 44:228-242, 1965; L. R. FEss, Tooth Eruption and Nutrition, $\mathrm{PhD}$ thesis, Tulane University, 1965). It is reasonable, therefore, to consider the value of tooth formation as a prenatal reference standard by first testing its validity at various developmental horizons.

We used nearly 5,0005 to 15 micrometer $(\mu \mathrm{m})$ histologic jaw sections of 52 first-trimester human embryos in the 14 to $58 \mathrm{~mm}$ crownrump (C-R) range. The data for 440 deciduous teeth from 22 male embryos were separated from the data for 600 deciduous teeth from 30 female embryos. A series of eight "stages" of dental development was used (cf A. R. Burdi, S. M. GARN and R.L. Miller, $J$ Dent Res 49:644, 1970) for both left and right teeth. Dental-stage data, C-R length data, and restricted gestational age data (based on last menstrual periods) were stored on standard 80-column IBM punch cards.

Since preliminary analysis of the relationships between $C-R$ length and tooth stage showed linear relationships for each of the 20 deciduous teeth in both sexes, computation of correlation coefficients was appropriate and values of $r$ for left and right sides could be pooled. As shown in the table, Pearsonian correlation coefficients for tooth stage and C-R length were uniformly high, for all classes of teeth and for both sexes, averaging 0.90 overall. The relationship between $C-R$ length and tooth stage proved systematically higher for

This study was supported in part by Grants HD02272 and University of Michigan Facuity Research Grant Project 21.

Received for publication February 9, 1970.

Additional information available on request to authors. female embryos than for male embryos: 10:0 against the $5: 5$ or chance bypothesis, a difference significant at any reasonable level of confidence. A separate procedure, which consisted of averaging tooth stages for all 20 deciduous teeth for each specimen, proved practical and yielded slightly higher computer-calculated correlation coefficients (Table). In contrast, correlations involving estimated gestation length were slightly lower, approximating 0.90 for females and 0.82 for males.

These exploratory findings for first-trimester prenatal dental development indicate the value of dental status as a reference standard comparable to, but separate from, the traditional parameter of C-R length. The results show, moreover, a systematic sex difference with higher tooth-stage, body-size correlations for females than for males, which is true in many aspects of postnatal ossification, eruption, and movement. Individual deviation from the general trend may further indicate abnormalities of fetal development and identify teratologies not evident by gross inspection or routine histological examination.

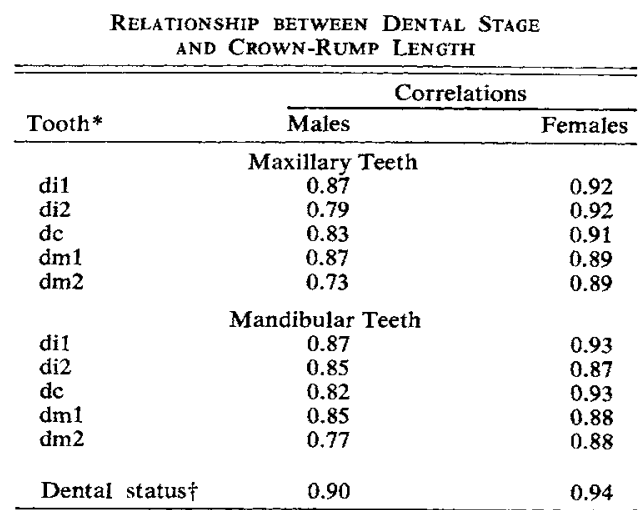

* $\mathrm{i}$, deciduous incisor; $\mathrm{c}$, deciduous canine; $\mathrm{dm}$, deciduous molar.

$\dagger$ Average dental stage for all 20 deciduous teeth. 